\title{
FORUM
}

\section{An Abacus for Windward Performance}

\section{J. Elliot}

THE prudence of determining the best windward and running courses for sailing yachts can be seen from Fig. $I$ in which the heart shaped curve represents a polar diagram of yacht performance with respect to the true wind direction. It is a diagram of yacht velocity $V$ and the angle $\alpha$ between the yacht's track and the true wind direction. The gap in the curve dead to windward is an area over which no performance is possible, whereas any destination lying outside this zone can be reached directly; there is only one sailing angle at which a destination lying between $X$ and $Y$ can be reached the quickest. A similar situation occurs on the

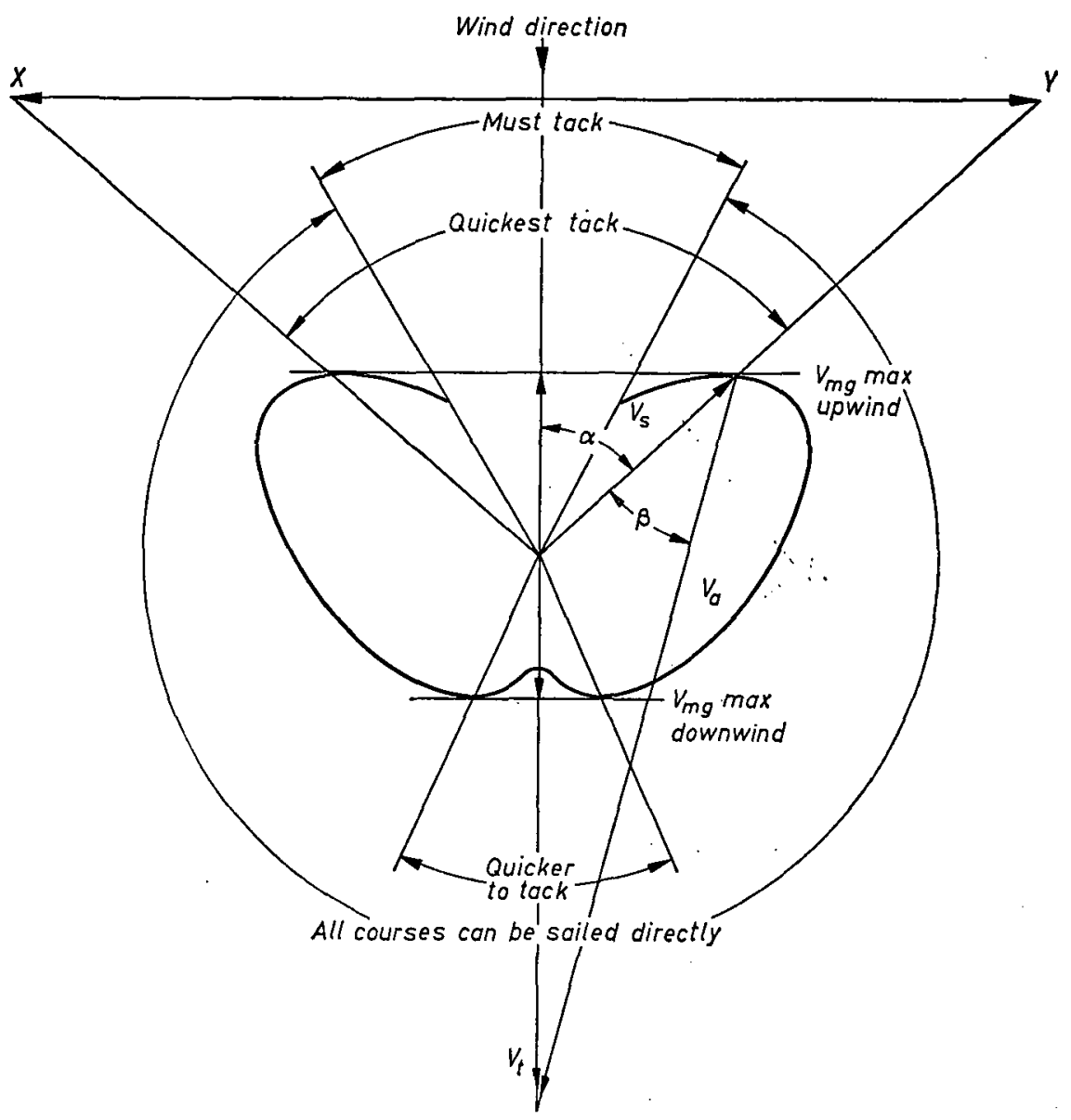

FIG. I. Polar diagram of sailing performance 
running point of sailing where the curve may have an inflexion, i.e. there is profit in tacking a course downwind.

The rate of progress to windward for any sailing angle and velocity may be assessed by resolving the yacht velocity along the true wind vector. The angle at which this resolved velocity $\left(V_{\mathrm{mg}}\right)$ reaches a maximum yields the quickest course. From the trigonometry $V_{\mathrm{mg}}=V_{\mathrm{s}} \cos \alpha$. Although the angle $\alpha$ cannot be measured on board, due to the forward motion of the yacht, it can be deduced from other measurable parameters, the apparent wind angle $\beta$ and the apparent wind speed $V_{\mathrm{a}}$. These parameters may be measured by masthead equipment, but some kind of computation must be made to deduce $\alpha$, and hence $V_{\text {mg }}$, from $V_{s}, V_{\mathrm{a}}$ and $\beta$.

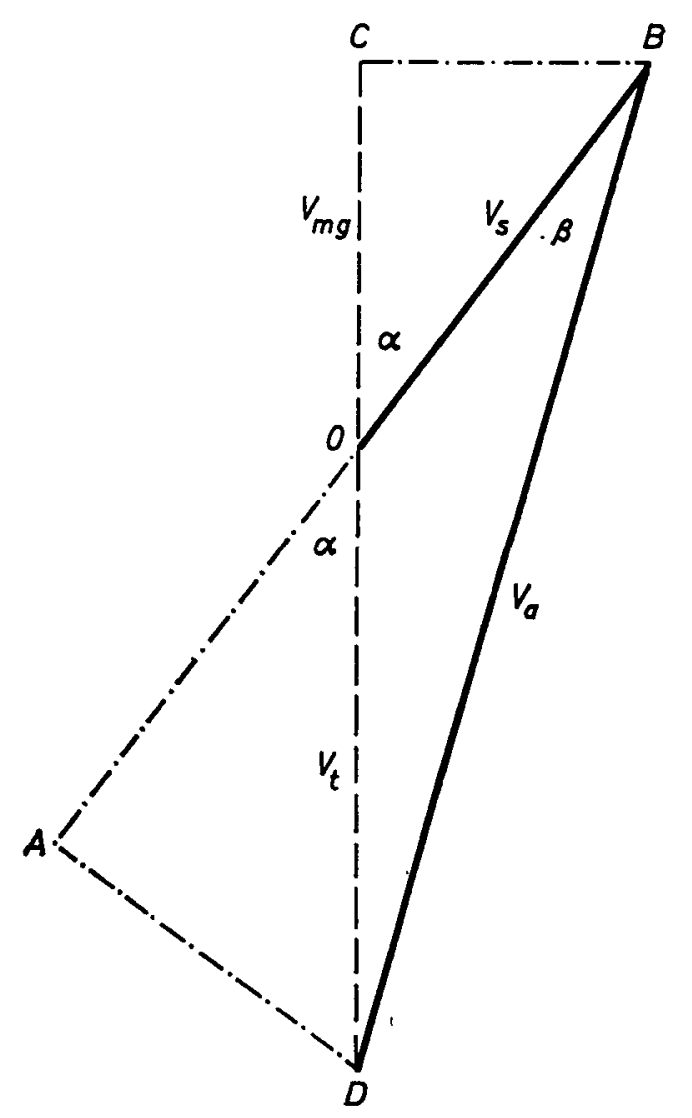

FIG. 2. Resolution of velocity vectors

In Fig. 2 the measured parameters are shown in solid lines and the developed parameters in dashed lines; the pecked lines complete the right-angled triangles. In the triangle $\mathrm{BAD}$ :

$$
\cos \alpha=\left(V_{\mathrm{a}} \cos \beta-V_{\mathrm{s}}\right) / V_{\mathrm{t}}
$$

and from the original equation:

$$
V_{\mathrm{mg}}=\left(V_{\mathrm{a}} V_{\mathrm{s}} \cos \beta-V_{\mathrm{s}}^{2}\right) / V_{\mathrm{t}}
$$


This however still contains the unmeasurable parameter $V_{t}$, which can be found by using the cosine rule $c^{2}=a^{2}+b^{2}-2 a b \cos C$, so that:

$$
V_{\mathrm{t}}^{2}=V_{\mathrm{a}}^{2}+V_{\mathrm{s}}^{2}-2 V_{\mathrm{a}} V_{\mathrm{s}} \cos \beta
$$

The solution using measured parameters is therefore:

$$
V_{\mathrm{mg}}=V_{\mathrm{a}} V_{\mathrm{s}} \cos \beta-V_{\mathrm{s}}^{2} /\left(V_{\mathrm{a}}^{2}+V_{\mathrm{s}}^{2}-2 V_{\mathrm{a}} V_{\mathrm{s}} \cos \beta\right)^{1 / 2}
$$

This equation is obviously too unwieldy for on-board measurements to be taken and solutions calculated. To realize a graphical solution the four terms of the equation must be reduced to three by pairing off the velocities $V_{\mathrm{mg}} / V_{\mathrm{t}}$ and $V_{\mathrm{s}} / V_{\mathrm{a}}$. To produce the appropriate graphs the boundaries of interest must be declared, for which purpose the following points have been taken into account.

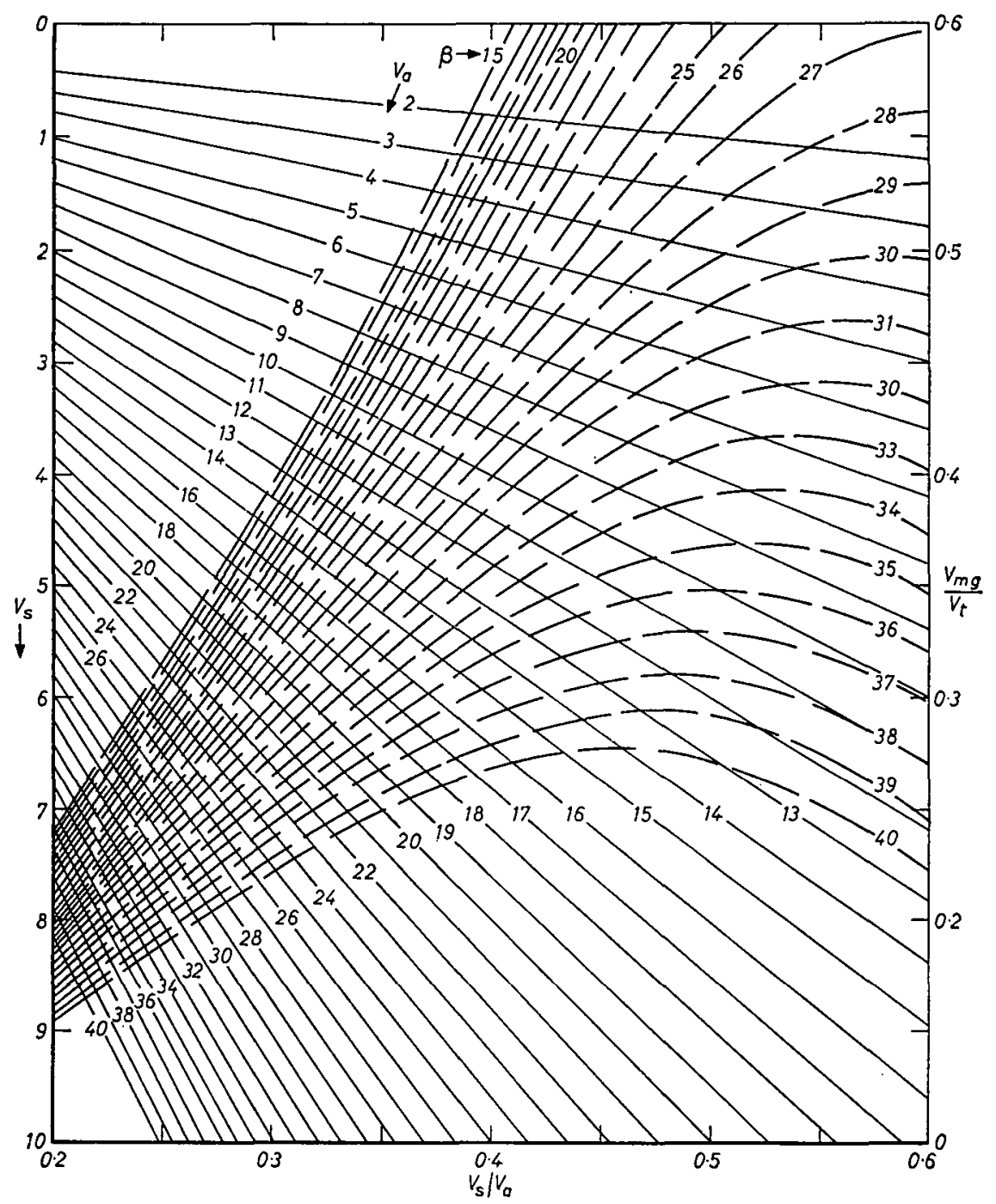

FIG. 3. The Abacus 
A yacht is unlikely to sail at speeds in excess of $0.6 V_{\mathrm{a}}$ and the maximum values of $V_{\mathrm{mg}}$ will occur within the apparent wind angles of $15^{\circ}$ to $40^{\circ}$, the corresponding cosines of which are 0.966 and 0.766 . $V_{\mathrm{mg}} / V_{\mathrm{t}}$ can never reach unity (by sailing directly into the eye of the wind) and even 0.6 is unlikely to be attained. On this basis the curves for $\beta$ from $15^{\circ}$ to $40^{\circ}$ are shown in Fig. 3 .

To apply the curves a known practical polar diagram, Fig. 4, for a true wind velocity $V_{t}$ of $11.5 \mathrm{kt}$., can be considered. The relevant values are shown in Table $I$ and the curves for each parameter in Fig. 5, from which it will be seen that the $V_{\mathrm{mg}}$ curve has a maximum almost coincidental with the maximum of $V_{\mathrm{a}}$. This feature will be dealt with later but, in general, the apparent wind velocity will maximize slightly further off the wind than does the curve of $V_{\text {mg }}$. This provides a ready guide to the vicinity of the $V_{m g}$ maximum and a course steered to maximize $V_{\mathrm{a}}$ is a logical starting point.

In the example this will be in the region where $\beta$ is between $24^{\circ}$ and $26.5^{\circ}$, and a course steered at the former angle under steady wind conditions would indicate $V_{\mathrm{a}}=18 \cdot 4$ and $V_{\mathrm{s}}=8$. The entry point on the abacus will be at $V_{\mathrm{s}}=8$

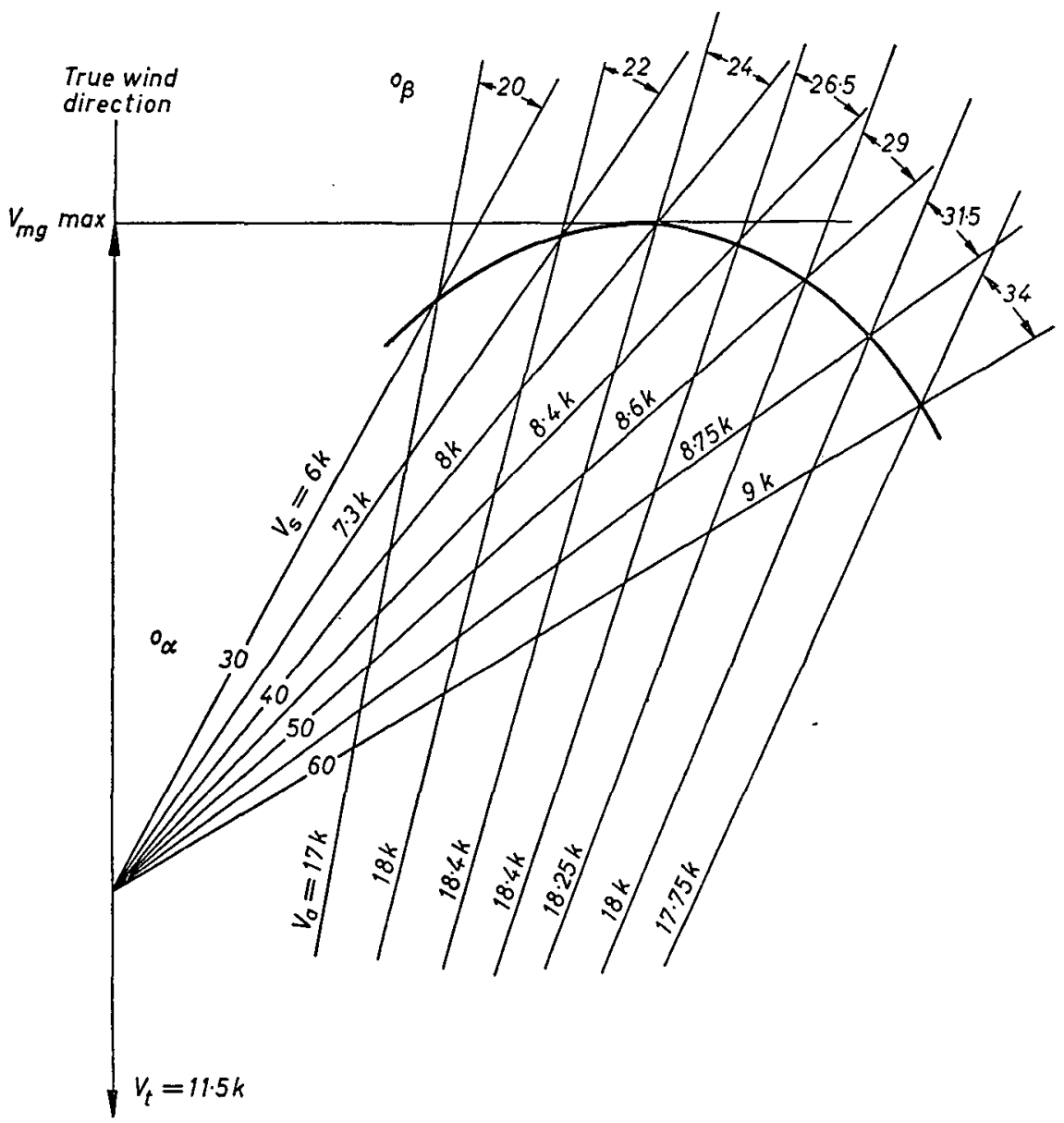

FIG. 4. Typical polar diagram (upwind) 
NO. 2

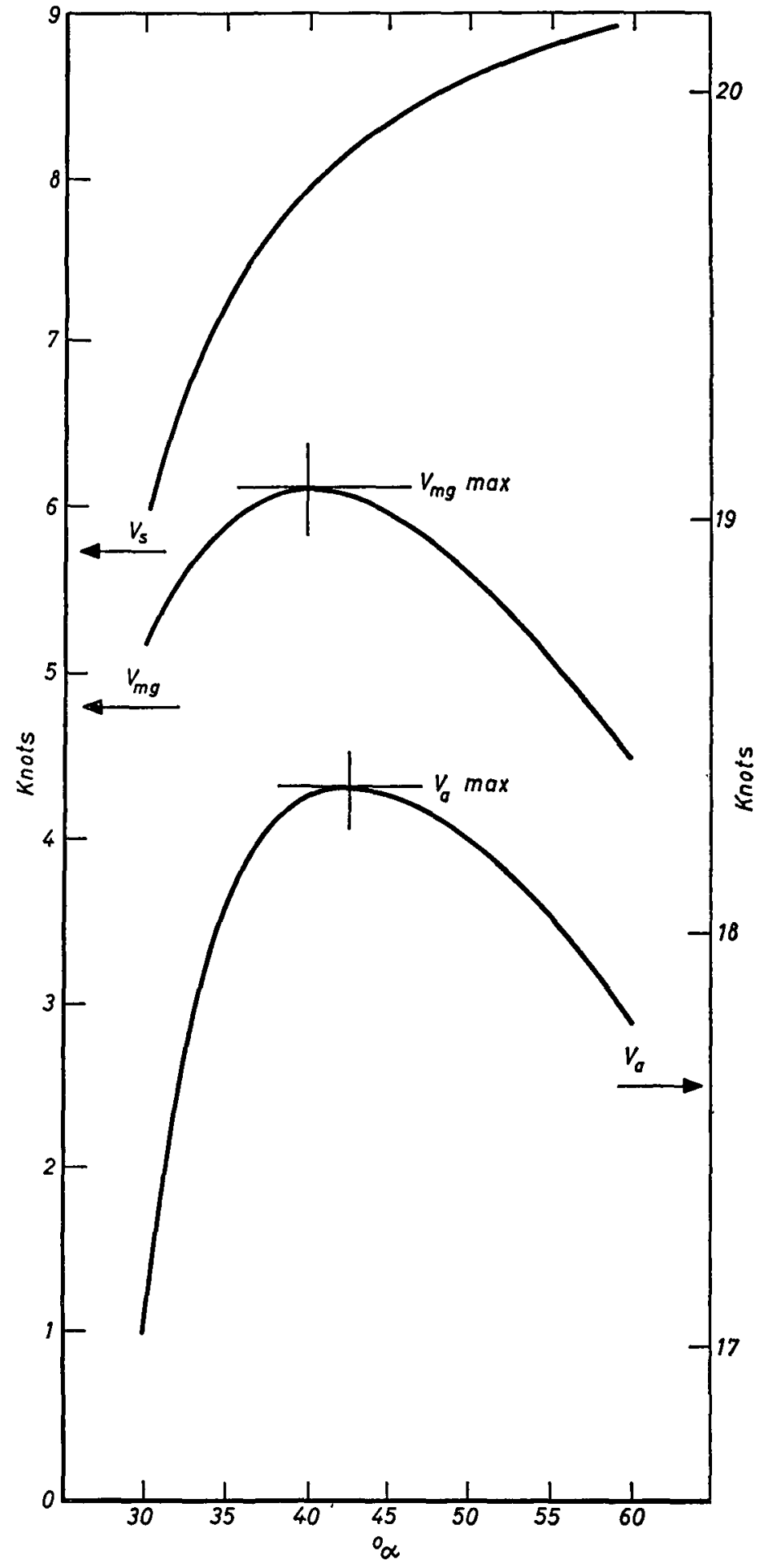

FIG. 5. Upwind polar plots 
TABLE I

\begin{tabular}{l|l|l|l|l|l|l}
\hline$\alpha$ & $\beta$ & $V_{\mathrm{a}}$ & $V_{\mathrm{s}}$ & $V_{\mathrm{s}} / V_{\mathrm{a}}$ & $V_{\mathrm{mg}}$ & $V_{\mathrm{mg}} / V_{\mathrm{t}}$ \\
\hline 30 & 20 & 17 & 6 & 0.354 & 5.196 & 0.450 \\
35 & 22 & 18 & 7.3 & 0.405 & 5.986 & 0.512 \\
40 & 24 & 18.4 & 8 & 0.435 & 6.128 & 0.530 \\
45 & 26.5 & 18.4 & 8.4 & 0.456 & 5.880 & 0.512 \\
50 & 29.5 & 18.25 & 8.6 & 0.470 & 5.500 & 0.476 \\
55 & 31.5 & 18 & 8.75 & 0.485 & 4.990 & 0.434 \\
60 & 34 & 17.75 & 9 & 0.505 & 4.50 & 0.390 \\
\hline
\end{tabular}

on the left-hand vertical axis. This value is now traced horizontally until the relevant $V_{\mathrm{a}}$ curve (straight lines running diagonally downward from left to right) is met, and from this point tracing either up or down vertically until the corresponding $\beta\left(=24^{\circ}\right)$ curve is encountered, to find $V_{\mathrm{mg}} / V_{\mathrm{t}}$ (i.e. at 0.53 ). Trial courses at $\beta=22^{\circ}$ and $26^{\circ}$ will result in lower values of $V_{\mathrm{mg}} / V_{\mathrm{t}}$, indicating that the best course has been found first time.

As with any practical method of tackling the problem there is the difficulty of reading the three dials simultaneously, and the method invites instrumental

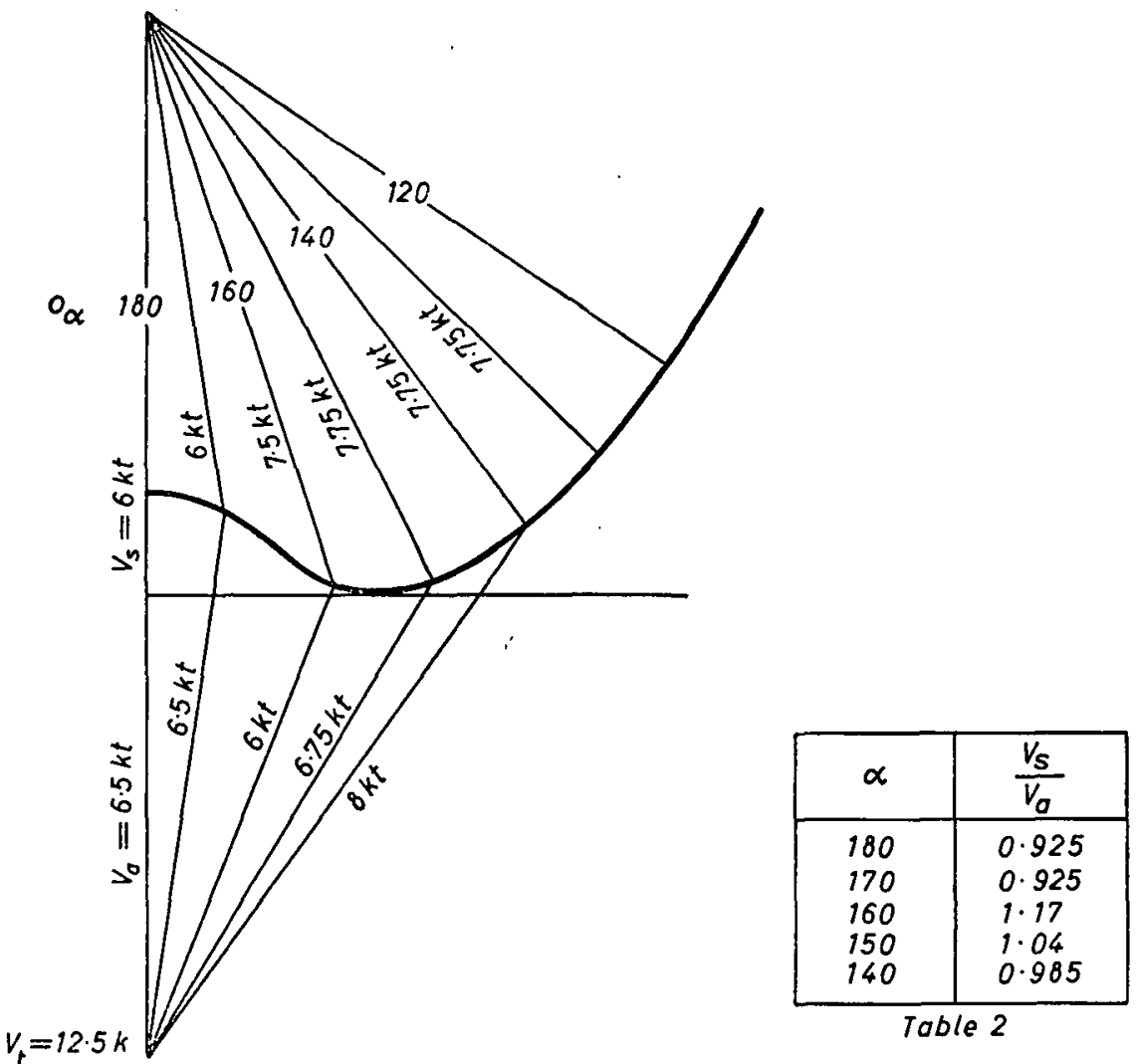

FIG. 6. Typical polar diagram (downwind) 
refinement by providing sample and hold facilities. By this means the angle meter may be watched for coincidence with the trial value and, when steady, the $V_{\mathrm{a}}$ and $V_{s}$ values may be held for say $20 \mathrm{sec}$. while the readings are noted.

Mention was made earlier of the proximity of the $V_{\mathrm{a}}$ and $V_{\mathrm{mg}}$ peaks and it follows from the geometry that the higher the wind velocity, the closer the peaks become. At what point this occurs in the wind scale depends upon the shape of the polar curve and the size of the yacht. It has been observed that up to the point where the maximum velocity for the yacht has been realized the $V_{\mathrm{mg}} / V_{\mathrm{t}}$ curve is linear and that beyond this point performance apparently tends to fall off. The point where the $V_{\mathrm{a}}$ meter alone may be used to indicate the best course will have to be a matter of individual subjective determination. That the effect does occur can be seen from a manipulation of the $V_{\mathrm{mg}} / V_{\mathrm{t}}$ equation, which may be re-written :

$$
\left(2 V_{\mathrm{mg}} / V_{\mathrm{t}}\right)+\mathrm{I}=\left(V_{\mathrm{a}}^{2}-V_{\mathrm{s}}^{2}\right) / V_{\mathrm{t}}^{2}
$$

from which it will be seen that, as $V_{\mathrm{a}}$ becomes larger and $V_{\mathrm{s}}$ attains its maximum value, $V_{s}$ cannot substantially alter the angle $\alpha$ at which $V_{\mathrm{mg}}$ becomes a maximum, i.e. when $V_{\mathrm{a}}$ also becomes a maximum. In the downwind case, where the polar curve may have an inflexion as shown in Fig. 1 , the maximum value of $V_{\mathrm{mg}}$ occurs when $V_{\mathrm{s}} / V_{\mathrm{a}}$ is at a maximum. How this happens will be readily seen from Fig. 6 which is an enlarged portion of the downwind area.

In conclusion it must be said that the $\beta$ meter should have a scale capable of indicating $\mathrm{I}^{\circ}$ increments. The other two velocity instruments could profitably be repeating instruments with sample and hold facilities. The $V_{\mathrm{a}}$ repeater would also benefit the user if an expansion (i.e. non-linear) amplifier, with offset and a qualitative scale, could be brought into commission once the sailing conditions warrant its use as a $V_{\mathrm{mg}}$ meter.

The author is grateful to the Board of EMI Marine for permission to publish this work.

\title{
The Use of ABC Tables in Preparing for Star Observations
}

\author{
Captain P. A. Thompson
}

As part of their navigational outfit many of the ships in service today are equipped with Sight Reduction Tables (A.P. $3270=$ H.O. 249), and many navigating officers include amongst their personal kit a Rude Star Identifier (H.O. 2102), or some similar device according to individual preference, for the purpose of obtaining the approximate altitude and azimuth of a selected body as a preliminary to making observations, particularly for star sights. However, for the benefit of those without such means at their disposal and as a matter of interest for others, the use of $A B C$ tables for this purpose is suggested. The origin and author of this method are not known and it does not appear to be included in any of the navigational manuals which have been in circulation in recent years, but there 